\title{
¡FULL LINDO!: ACERCA DEL USO DE FULL EN EL HABLA DE ECUADOR
}

\section{Ana Estrella-Santos ${ }^{1}$}

\section{RESUMEN}

Full es un anglicismo ampliamente usado en español, especialmente en expresiones como full-time y full-equipped. Otros usos, sin embargo, están restringidos diatópica y diastráticamente. El objetivo de este trabajo es analizar todos los usos de full en el habla de Ecuador, poniendo especial énfasis en su uso como intensificador (full linda y full bien) que es el menos estudiado. Para ello, se han hecho 45 encuestas directas a informantes quiteños de entre 18 y 26 años, se han analizado dos horas de grabaciones a jóvenes del mismo rango de edad, se ha hecho una búsqueda sistemática de ejemplos en Internet, y se han utilizado los corpus de la Academia de la Lengua para constatar los diferentes usos a lo largo de Hispanoamérica. Este estudio ha encontrado que el uso de full, en el registro informal, es general entre los jóvenes de Ecuador. Además, en el caso de las perífrasis de intensificación, full está reemplazando a bien, y, en esos contextos, full no pierde su significado inicial de 'lleno'y, por lo tanto, se evitan las expresiones como "full poco" o "full vacío". Desde el punto de vista pragmático, este uso de full funciona como distintivo del habla juvenil. Los otros usos -"a full" como locución adjetival y full como adjetivo- son también propios de la oralidad y del registro informal pero el rango de edad de quienes lo usan frecuentemente es mayor entre 18 y 54 años.

Palabras clave: intensificador, full, habla del Ecuador, dialectología, pragmática, anglicismo

\section{ABSTRACT}

Full is an Anglicism widely used in Spanish, especially in expressions like full-time and full-equipped. Other uses, however, are restricted geographically and diastratically. The aim of this paper is to analyze all the uses of full in Ecuadorian Spanish, with particular emphasis

1 Pontificia Universidad Católica del Ecuador, Facultad de Comunicación, Lingüística y Literatura, Quito, Ecuador (atestrellla@puce.edu.ec). 
on its use as an intensifier (full linda and full bien) that has not been thoroughly studied. To this end, we have: a) analyzed 45 surveys that were given to quiteños between 18 and 26 years old; b) examined two hours of recordings of subjects of the same age range; c) explored the Internet to look for examples of full; and d) researched the Corpus of the Academy of Spanish Language in order to verify the different uses of this word throughout Latin America. This study determined that young Ecuadorians are using full in the informal register. In addition, when full is used as an intensifier in periphrasis, full is replacing bien, and, in these contexts, full does not lose its original meaning of 'having no empty space' and, therefore, expressions as "full poco" or "full vacío" are rarely used. Pragmatically, this use of full functions as a distinctive trait within urban youth speech. Other uses - " a full" and full as an adjective - are also characteristic of spoken and informal register but the age range of those who frequently use it is broader: between 18 and 54.

Key words: intensifier, full, Ecuadorian Spanish, dialectology, pragmatics, Anglicism.

\section{INTRODUCCIÓN}

El objeto de este trabajo es analizar full en el habla de Ecuador. Para ello se describen sus usos en expresiones como full-time y full-equipped, como adjetivo, en locuciones adjetivales y adverbiales y como intensificador en perífrasis adjetivales y adverbiales (full linda y full bien). Todos estos aspectos se analizarán en este artículo; así, en primer lugar, se describirán su extensión y su uso a lo largo de Hispanoamérica. Después, se explicarán los usos en las locuciones adjetivales y cuando funciona como un adjetivo. Por último se estudiarán las características semánticas de su uso como intensificador, que es un fenó- meno relativamente nuevo y poco estudiado, descrito en el Diccionario de Americanismos (DA) para Ecuador, Colombia, Venezuela y Perú. Hay que apuntar que en el Corpus del Nuevo Diccionario Histórico del Español (CDH), full se encuentra ya en 1908, en una publicación de Perú ("pasara un auto a todo full"), y, aunque los diversos usos aquí descritos, solo aparecen a partir de la década del 80 del siglo pasado, podemos considerar que full en el español no es un fenómeno reciente, aunque como veremos, algunos de sus usos, sobre todo con el significado de'muy'y'mucho', sí lo son. 


\section{MATERIALES Y MÉTODOS}

Para la recogida de datos se hizo teniendo en cuenta cuatro métodos: a) 45 encuestas directas a 23 hombres y 22 mujeres que viven en Quito y cuyas edades oscilan entre 18 y 26 años. En estas encuesta se preguntó específicamente si usaban o no full y cómo lo usaban. Se pidió además un ejemplo². b) Un análisis de 2 horas de grabaciones. Estas grabaciones se hicieron con sujetos de edades similares (18 a 26 años). Aquí se les pidió que hablaran en grupo libremente. c) La búsqueda de ejemplos del uso de full en la red de Internet. Esta búsqueda no se centró solo en Ecuador sino también en otros países. d) La búsqueda en los diccionarios y corpus de la Academia: Diccionario de la Real Academia de la Lengua (DRAE), Diccionario de Americanismos (DA), Diccionario Panhispánico de Dudas (DPD), en el Corpus de Referencia del Español Actual (CREA), el Corpus del Español del Siglo XXI (CORPES) y el Corpus del Nuevo Diccionario Histórico del Español (CDH).

\section{EL USO DE FULL COMO PRÉSTAMO INTEGRAL}

El préstamo, entendido como la transferencia integral de un elemento léxico extranjero (Gómez Capuz, 2009), es un proceso frecuente de ampliación léxica. En Hispanoamérica y España se usan muchas expresiones con full, aun cuando su traducción podría ser muy sencilla. Por ejemplo, full-time, recogida en el DRAE, cuyo significado es"con dedicación exclusiva", no tiene ninguna marca diatópica, por lo cual se supone del español estándar. La primera vez que aparece en el CDH es en un texto de Bioy Casares, de Argentina, en 1962 ("quieren ocuparnos full time").
EI DPD añade, además, half-time ('con media jornada') y part-time ('con dedicación parcial o no exclusiva') y, aunque explica que hay formas equivalentes en español, no da consejos sobre su uso preferente. La Fundéu (2016) en cambio sí recomienda sus correspondientes en español. Esto da cuenta de la extensión de estas expresiones.

Otros préstamos similares que hemos encontrado en Ecuador, pero que también son frecuentes en otros países latinoamericanos y en España son full equipped, full edition, full frame, full $H D$. En el CREA para Ecuador solo hay

\footnotetext{
2 Para evitar la distancia entre entrevistador e informante, estas encuestas fueron hechas por los estudiantes del cuarto semestre de la materia de Lingüística, de la carrera de Comunicación de la PUCE, entre marzo y mayo del 2014.
} 
tres ocurrencias de full: una en la que es parte del título de una película y las otras dos son: full color y full equipo (refiriéndose a completamente equipadas). En el CORPES, en una búsqueda sin restricción geográfica, full HD aparece 84 veces; full speed, full caché, full service, 10 veces cada uno. Hay también cuatro ocurrencias de préstamos parciales como, full equipado, full color, full de ases o de reyes (para el juego de cartas).

\section{La locución adjetival ${ }^{3}$ a full}

La locución a full es frecuente en gran parte de Hispanoamérica. Aunque el DA considera que la restricción diatópica es Ecuador, Perú, Bolivia, Argentina, Paraguay y Uruguay, una búsqueda sistemática en las redes sociales, permite constatar que hay ejemplos de otros países como México y Venezuela. Según el DA, semánticamente esta locución tiene dos acepciones: si se refiere a una persona significa "muy ocupada o atareada" y, si no, es sinónimo de 'lleno'.

La primera acepción podría considerarse como una ampliación semántica de la primera. En el inglés estándar, full como adjetivo significa 'lleno', pero no significa nunca'muy ocupado'. Algunos enunciados en inglés con este significado son: "I'm packed today", "Today is packed for me" o "Today is jam-packed".

En el CORPES hay ejemplos de Ar- gentina, Ecuador, México y Uruguay. En el corpus recogido de las encuestas y en las grabaciones encontramos "Estoy a full" (estoy muy ocupado); "La fiesta estaba a full" (La fiesta estaba llena/a rebosar).

\section{La locución adverbial a full}

Ninguna de las dos acepciones recogidas por el DA en el apartado anterior servirían para explicar el uso de esta locución (recogida en las entrevistas) cuando modifica a un verbo: "Hablamos a full". En este caso significa "hasta el límite de las fuerzas o posibilidades". En el CORPES también se encuentran algunos ejemplos: "apoyarnos a full", "vivir la democracia a full" (Argentina), "trabajar a full" (Chile), "parece estar trabajando a full" (Uruguay).

\section{Full como adjetivo}

El DA proporciona dos acepciones de full como adjetivo (además de 'mucho' que se describe en la siguiente sección). La primera es 'lleno' que tiene las siguientes marcas diatópicas: Estados Unidos, México, República Dominicana, Puerto Rico, Colombia, Venezuela, Ecuador, Perú, Chile y Bolivia. En nuestro corpus hemos encontrado "estoy full", "el vaso está full de agua", "está full de canguil".

\footnotetext{
${ }^{3}$ Las categorías gramaticales que se usan en este artículo son las que consigna el DA bajo el lema full.
} 
La segunda acepción del DA se considera solo para Ecuador: "referido a una habitación, completamente amueblada". Esta acepción realmente no parece ser general en nuestro país, a pesar de que esté en el DA. Se hicieron 50 encuestas para preguntar sobre este punto ninguno de los encuestados dijo que entendiera "la habitación está full" como "completamente amueblada". Todos consideraron que significaba que estaba 'repleta' o 'muy llena'. Una búsqueda exhaustiva en la internet tampoco dio cuenta de este uso.

\section{Full como 'mucho' y'muy'}

El DA considera que, como equivalente a'muy'y'mucho', solo se usa en Colombia, Venezuela, Perú y Ecuador. Además, diastráticamente lo restringe al habla juvenil. En el habla de Ecuador, aunque hay algunas personas de mediana edad (entre 35 y 45) que lo usan, lo normal es que sea una característica de los jóvenes. Nuestras encuestas fueron hechas en Quito, pero el análisis de las redes sociales nos permitió ver que es un fenómeno que se da en todo Ecuador. No hay una restricción geográfica.

Al igual que ocurre con 'mucho', full, antepuesto al sustantivo, actúa como adjetivo con el significado de 'abundante': "full agua en las calles", "full hambre", "full comida", "me dijeron que iban a ir full personas a esa fiesta","fueron full gente al concierto", "tengo full calor", "hay full tráfico", "tengo full deberes".

Full también equivale a mucho cuando funciona como adverbio, con el significado de 'abundantemente o intensamente': "me gusta full esta comida", "trabajamos full", "comí full". En el CORPES, aparte de los países señalados en el DA, hay ejemplos de México ("andamos full'").

Full antepuesto a los adjetivos y adverbios es similar a 'muy' y sirve para construir el grado superlativo: muy pequeño es equivalente a pequeñísimo. De la misma manera full pequeño significaría pequeño en un grado superlativo. Así, al igual que 'muy', modifica la intensidad de la cualidad denotada por el adjetivo (full linda/muy linda) y cualifica a los adverbios (full bien/muy bien).

Cuando está antepuesto a los adjetivos, convive también con la perífrasis bien + adjetivo. Hay pues, tres formas para intensificar el valor de los adjetivos:

$$
\begin{aligned}
& \text { muy + adjetivo: muy linda } \\
& \text { bien + adjetivo: bien linda } \\
& \text { full + adjetivo: full linda }
\end{aligned}
$$

Entre estas tres formas no hay una alternancia libre, puesto que se usa en registros diferentes. También, desde el punto de vista semántico hay matices que los hacen distintos. 
Las diferencias entre muy y bien en perífrasis con un adjetivo han sido extensamente analizadas por Serradilla Castaño (2006) quien considera que la perífrasis bien + adjetivo es utilizada sobre todo en el habla coloquial, en lengua oral y"preferentemente por las mujeres $y$, dentro de este grupo, entre las de un nivel cultural más bajo, es decir, en aquellos casos en los que la presión de la norma es más débil" (p. 218). Puesto que no tenemos un estudio similar en Ecuador, consideramos que estos datos coinciden con lo que intuitivamente vemos en el habla ecuatoriana, aunque habría que contrastar con lo que Serradilla Castaño (2006) apunta en relación con el estrato de edad, quien encuentra que los jóvenes de entre 18 y 34 usan más la perífrasis bien + adjetivo que los de la generaciones mayores. En Ecuador, entre los jóvenes de niveles culturales bajos y altos, y especialmente en las ciudades, hay una tendencia a preferir la forma full + adjetivo como superlativo. La forma bien+ adjetivo se usa menos en este socioestrato. Esta perífrasis tiene una restricción de registro, ya que se considera coloquial o informal. De hecho, algo que la autora ha observado es que durante las exposiciones de los estudiantes universitarios (de edades entre 18 a 22 años), cuando alguien decía sin querer full ("esta parte es full interesante", "fue full difícil encontrar información"), el resto de la clase se reía ya que encontraba chocante este uso en una situación formal.
En las entrevistas se preguntó sobre el grado de intensificación. Las respuestas siempre fueron que full + adjetivo tenía un grado mayor que muy + adjetivo y que muchos no decían nunca bien + adjetivo. Algo similar ocurre cuando va antepuesto a un adverbio (full bien): funciona como muy, pero tiene la restricción de registro (es informal) y de edad (lo usan mayoritariamente los jóvenes). Al igual que ocurre con 'muy', full puede reduplicarse para intensificar su sentido "full, full rápido".

En los dos casos en los que es equivalente a muy (como intensificador de un adjetivo," full mala" o de un adverbio, "full mal") hay un matiz adicional. Veamos los siguientes ejemplos:
(1)Ya no deseo más, estoy full lleno
(2) El concierto estuvo full bien
(3) Full bacán
(4) Esa cosa está full berreada
(5) Full mal
(6) Full poco
(7) Full escaso
(8) Full vacío

Del análisis de las respuestas dadas en las 45 encuestas y de las grabaciones, se desprende que, en general, full, cuando modifica a los adverbios o adjetivos, tiene una connotación de cantidad positiva. Es decir, a diferencia de muy que es neutra en este sentido, full no suele ser el intensificador de adver- 
bios o adjetivos que signifiquen una pequeña o nula cantidad. Por ejemplo, en (6), (7) y (8) dos informantes explicaron que no usarían esas formas porque no tiene ninguna relación con el significado de full. Para uno de ellos, este tipo de uso era "ignorante". Otra informante explicó que una vez dijo full poquito y se sintió mal. Estas respuestas salieron espontáneamente, sin que hubiera una pregunta específica acerca de este uso restringido. Sin embargo cuando se hizo una encuesta específica con 50 informantes, el 90\% explicó que nunca usarían las formas (6), (7) y (8). En relación con otras connotaciones como características positivas (linda, interesante, fácil) o negativas (difícil, feo, asqueroso), el uso es indiferente.

Esta restricción indica que full mantiene su valor semántico de'lleno'que es lo que significa en inglés. De hecho, el Merriam Webster Dictionary (n.d.), apunta que full viene del antiguo germánico fol, del latín plenus, y este del griego plèrēs. En inglés puede funcionar como el adverbio very (muy): They knew full well they had lied to me, pero incluso en esto casos, el sentido de completamente, no se ha perdido. Además es una forma que funciona en frases hechas, generalmente con well. En el habla estándar se prefiere el adverbio fully. Full no se usa en enunciados como full difficult, full good.

\section{¿Por qué se usa full?}

Dejando de lado el préstamo integral en expresiones que se transfieren directamente del inglés del tipo full-time, y que no tienen restricción de registro ni de edad, los otros usos descritos en este artículo se consideran coloquiales y propios de la oralidad. La perífrasis a full y full como 'lleno' tienen un uso más extendido y en relación con la edad ya que es común entre los de 18 a 34 y los de 35 a 54. Es menos usada a partir de los 55.

La revisión de las grabaciones del habla juvenil de Quito permitió ver que full reemplaza a bien. No hubo en el corpus analizado ningún caso en el que se usara bien como perífrasis de intensificación, pero sí hubo ejemplos del uso de muy. El uso como intensificador (ya sea como adjetivo o como adverbio) tiene, por lo tanto, una restricción muy clara en relación con la edad. Es difícil analizar la necesidad de esa intensificación. ¿Qué le aporta al hablante full que no tengan ya bien o muy? Sin duda, full actúa como un distintivo del habla juvenil. Un 73\% de los entrevistados consideró, además, que este era un uso propio de los ecuatorianos. Aunque, como hemos visto, esto no es verdad, desde el punto de vista pragmático funciona también como una particularidad diatópica. Rodríguez (2002) citado por Jørgensen y López (2007) considera que el habla juvenil se caracteriza por "una selección 
que tiene que ver con el distinto grado de inseguridad y competencia lingüística en los grupos de edad inferior. Así son los jóvenes los que exhiben un mayor empleo de muletillas y un estilo verbal menos cualitativo" (p. 2). La inse- guridad lingüística, por lo tanto, crea un espacio para identificarse como grupo y marcar las diferencias con las generaciones mayores. Además, está el hecho de que el uso de vocablos en inglés tienen una marca positiva (Zimmerman, 2002).

\section{CONCLUSIONES}

El uso de full es general entre los jóvenes de Ecuador. Aunque algunos usos como los préstamos integrales o la locución a full, se empezaron dar en las personas que actualmente tienen entre 30 y 50 años; los otros usos, especialmente como intensificador, son más recientes. Este no es un fenómeno exclusivo del Ecuador y su uso pudo haberse propagado desde otros lugares como Colombia que goza de una apreciación lingüística positiva. Además, la televisión puede haber jugado un papel importante en esta irradiación, debido a la gran cantidad de programas colombianos que se trasmiten en los canales abiertos de Ecuador. El análisis que se ha realizado ha permitido ver los diferentes significados y usos que se da a full. Además, se ha visto que, en el caso de las perífrasis de intensificación, el full está reemplazando a bien. Además, es importante resaltar que, en esos contextos, full no pierde su significado inicial de 'lleno' $y$, por lo tanto, se evitan las expresiones "full poco" o"full vacío". Puesto que el uso como intensificador es relativamente reciente, es difícil saber si se trata de un fenómeno efímero o si, como en los otros casos (full como lleno y la locución a full), se va mantener como una marca coloquial y oral del español de ciertas regiones. 


\section{BIBLIOGRAFÍA}

Asociación de Academias de la Lengua Española (2010). Diccionario de americanismos. Madrid: Santillana. Disponible en http://lema.rae.es/damer

Fundéu BBVA - Fundación del español urgente (2015). Disponible en http:// www.fundeu.es/

Gómez Capuz, J. (2009, julio). El tratamiento del préstamo lingüístico y el calco en los libros de texto de bachillerato y en las obras divulgativas. TONOS. Revista Electrónica de Estudios Filológicos 17. Disponible en https://www.um.es/tonosdigital/znum 17/secciones/tritonos-1-librosdetexto.htm

Jørgensen, A. M. y López, J.A. (2007, agosto). Los marcadores del discurso del lenguaje juvenil de Madrid. Revista Virtual de Estudos da Linguagem - ReVEL. 5 (9). Disponible en www.revel.inf.br

Merriam Webster Dictionary (n.d.). Disponible en: http://www.merriam-webster .com/dictionary

Real Academia Española (2014). Corpus del nuevo diccionario histórico del español $(\mathrm{CDH})$. [en línea]. Disponible en: http://www.rae.es.
Real Academia Española (2014). Banco de datos (CORPES XXI), Corpus del español del siglo XXI [en línea]. Disponible en: http://www.rae.es/recursos/banco dedatos/corpes-xxi.

Real Academia Española (2014). Banco de datos (CREA). Corpus de referencia del español actual [en línea]. Disponible en: http://www.rae.es.

Real Academia Española (2014, 23a ed.). Diccionario de la lengua española (DRAE). Madrid: Espasa-Calpe. Disponible en http://lema.rae.es/

Serradilla Castaño, A. (2006). "BIEN" + adjetivo como perífrasis de superlativo en español. Particularidades semánticas y sintácticas. Verba 33: 215-233. Disponible en http://hdl.handle.net/10347/ 3461

Zimmermann, K. (2002). La variedad juvenil y la interacción verbal entre jóvenes. En: F. Rodríguez. El lenguaje de los jóvenes, Barcelona: Ariel. 